\title{
Knowledge related to nutrition and hypertension management practices of adults in Ga-Rankuwa day clinics
}

\author{
NG Nkosi, M Tech Nursing student \\ Adelaide Tambo School of Nursing Science, Tshwane University of Technology \\ SCD Wright, \\ Associate Professor, Adelaide Tambo School of Nursing Science, Tshwane University of Technology
}

\section{Kev words}

Hypertension, knowledge, nutrition, practices, management, Ga-Rankuwa

\begin{abstract}
Curationis 33 (2): $33-40$
Hypertension is a global, non-communicable chronic disease being asymptomatic and known as the silent killer with signs and symptoms only occurring when a target organ is damaged. Being a condition common in South Africa, hypertension is also a risk factor for cerebro-vascular incidents, myocardial infarction, left ventricular hypertrophy, renal disease and retinopathy. Black adults in an urban environment appear to be especially vulnerable to excessive increases in blood pressure. The research question explored was what was the knowledge of hypertensive adults attending day clinics in Ga-Rankuwa regarding nutrition and hypertension management practices. An exploratory strategy was used as no similar research had previously been conducted in Ga-Rankuwa. A cross sectional survey design was used to investigate hypertensive adults attending the three primary health clinics in Ga-Rankuwa. The sampling method was convenient and the sample size 101 participants. Two data gathering methods were used, these being physical measurements and self-report. For the self-report, a structured interview was conducted. The data were analysed using descriptive statistics. The results indicated a lack of knowledge regarding nutrition and management of hypertension. The proportion of participants with uncontrolled hypertension was high (58.6\%) and non-compliance with medication occurred frequently (58.1\%). A third (28.7\%) of the sample lacked knowledge of the complications of hypertension (28.7\%). A community-based intervention, based on the results of the study, is recommended.
\end{abstract}

\section{Correspondence address}

SD Wright

Private Bag X680

Pretoria

0001

Fax (012) 3825033

Work (012) 3825470

Mobile:0828211186

Email: wrightscd@tut.ac.za 


\section{Introduction}

Hypertension is a global, non-communicable chronic disease and is largely asymptomatic. Known as the silent killer, a person experiences very few signs and symptoms until damage occurs to a target organ. Hypertension is a common condition in South Africa and is a risk factor for cerebrovascular incidents, myocardial infarction, left ventricular hypertrophy, renal disease and retinopathy (Steyn, 2005:80). In 2000 in South Africa, non-communicable diseases caused $37 \%$ of deaths and $21 \%$ of premature mortality (Ijumba, Day \& Ntuli, 2004:176). Van Rooyen, Huisman, Eloff, Laubsher, Malan, Steyn and Malan (2002:69) report that black adults in an urban environment appear to be especially vulnerable to excessive increases in blood pressure, leading to hypertension in later life. In support, Peltzer (2001:52) reported that of the estimated 5.5 million people in South Africa with elevated blood pressure, 3 million are black males. Hypertension however, does not develop in isolation, as several risk factors are associated with hypertension.

Besides being a heredity trait, obesity, unhealthy diet, diabetes mellitus, excessive alcohol intake, physical inactivity and smoking are specific risk factors associated with hypertension (Department of Health, 2003:1). Of these risk factors, diet, physical activity, alcohol consumption and smoking are risks related to the person's lifestyle. Obesity and diabetes mellitus are secondary risk factors associated with the lifestyle risks with diet being a key risk factor for hypertension (Ijumba, Day \& Ntuli, 2004:182). South Africa has experienced major changes in food consumption with traditional plant-based foods being replaced with a diet characterised by high fat, high sugar and energy dense with low fibre food. This diet is strongly associated with the development of chronic cardiovascular disease. If people's physical stature can be taken as a proxy for the quality of their diet in terms of cardiovascular risk, many South Africans are at risk. Approximately three of every ten men $(29 \%)$ and six of every ten women (56\%) in South Africa are overweight (Reddy, 2004:175). Due to the lifestyle related risk factors for hypertension, the control of hypertension cannot rely alone on pharmacological management.

The control of hypertension comprises both pharmacological and non-pharmacological management to prevent complications (Department of Health, 2003:1). The management of hypertension has a significant lifestyle adjustment component, which could be a barrier to normal blood pressure for an extended period. Weight gain, high salt intake, tobacco use, high alcohol intake and increased psychosocial stress can lead to poor management of hypertension (Bales \& Ritchie, 2004:406; Steyn, 2005:83).

According to Peltzer (2002:182), a healthy lifestyle is one in which individuals are aware of risks to their health and can make informed choices for maintenance. These choices include stopping smoking, consuming only three alcoholic drinks per day, weight reduction and regular exercise (Smith, 1999:3). In addition, a diet with low sodium, low fat and plenty of fresh fruit and vegetables is required. Risk awareness is an important factor linked with knowledge and health belief. Health behaviours are associated with the actual practice of the health beliefs (Peltzer, 2002:188). Health promotion strategies can be formulated and implemented if the health beliefs and knowledge are known. Knowledge about nutrition and the risk factors associated with different types of nutrition is crucial to health promotion. Without knowledge, patients usually discontinue their nutritional management of hypertension, making it difficult to control and manage the disease, the result being an increase in hypertension related complications with a concurrent increase in premature mortality and socio-economic consequences (Peltzer, 2002:188).

\section{Research Question}

In 2003, the Department of Nursing Science at Tshwane University of Technology conducted a community study in Ga-Rankuwa. The study revealed that more women (46.1\%) than men $(45.8 \%)$ were hypertensive and obesity was prevalent in women $(69.8 \%)$ as well as in men $(40.9 \%$ ) (Wright \& Ramukumba, 2008:72). Comparing these figures to the South African figures for 2004 where only $29 \%$ men and $56 \%$ women were found to be obese (Reddy, 2004:175-179), clearly indicated that an in-depth investigation in the community was needed.

The formulated research question for the study was therefore, what is the knowledge regarding nutrition and hypertension management practices of hypertensive adults attending primary health clinics in Ga-Rankuwa.

\section{Objective of the study}

The objective of the study was to determine the nutrition-related knowledge and hypertension management practices of hypertensive adults attending primary health clinics in Ga-Rankuwa.

\section{Theoretical framework}

The Health Promotion Model (Pender, Murdaugh \& Parsons, 2002:60), focusing on promoting personal health and wellbeing, is utilised as the theoretical foundation of the study. It consists of seven cognitive-perceptual factors and five modifying factors that predict and explain health behaviours.

The cognitive-perceptual factors are the importance of health, perceived control of health, definition of health, perceived heath status, perceived selfefficacy, perceived benefits and perceived barriers. The modifying factors are the demographic and biological characteristics, interpersonal influence, situational influences and behavioural factors.

\section{Research methods and design \\ Setting}

The study setting was Ga-Rankuwa. According to Statistics South Africa (2001), the total population of GaRankuwa was approximately 83900 with the total number of households 21100 . The majority in terms of socio-cultural groups was Setswana speaking people $(74 \%)$. More than half of the community $(56.8 \%)$ had an educational level of below Grade 10 with only $32.7 \%$ of the population employed.

\section{Design}

The study used a cross-sectional descriptive design, which is a non-experimental design examining data from a specific group, at one point in time (LoBiondo-Wood \& Haber, 2006:244). 
Population, sample and size

The population investigated were hypertensive adults, 18 years of age and older, residing in the Ga-Rankuwa area and attending primary health clinics in Ga-Rankuwa for hypertension management. According to Burns and Grove (2005:47), the population is described as all the elements that comply with specific criteria for inclusion in a given universe. Patients from all three primary health clinics were conveniently included in the study with a specific week being arranged with the clinic's management to gather data. Adhering to the statistician's advice at Tshwane University of Technology, at least 30 participants per clinic had to be included and the sample size was 101 $(n=101)$.

\section{Data gathering}

Two data gathering methods used in this study were self report and physiological measurement. A validated questionnaire was used to gather data relating to hypertension knowledge, habit and lifestyle changes, quality of life and care, life threats and clinical detail with personal medical history. Several physical measurements, blood pressure, weight and height, hip and waist circumference and physical activity were taken. Participants were informed by clinic managers of the process and procedures of the study and informed consent was obtained before participation. Trained field workers conducted the structured interviews with participants to prevent selection bias due to low literacy level.

To ensure validity and reliability of the data the following measures were implemented:

- $\quad$ Each participant's blood pressure was measured using a calibrated baumanometer and stethoscope.

- The height measurements were taken using a stadiometer and an electronic digital scale measured weight. The participant's body mass index (BMI) was calculated using the standard formula of weight in $\mathrm{kg} /$ height in $\mathrm{m}^{2}$

- $\quad$ Hip and waist measurement, in male respondents, were taken in the upright position, with the circumference being measured to the nearest 0.5 centimetre. For males, the circumference was measured at the level of the umbilicus at expiration and for females at the narrowest point between the rib cage and the iliac crest.

\section{Data analysis}

Descriptive statistics were used in the analysis, to allow the researcher to describe and summarise data (LoBiondoWood \& Haber, 2006:358). The SPSS programme (version 14) was used to analyse the data. As the data was not generated from a random sample, two non-parametric statistical tests were performed, a Chi-square test and the Pearson product moment correlation coefficient.

\section{Validity and reliability}

Several measures were taken to ensure data validity and reliability.

- The hypertension diagnosis was confirmed from the patient's file before they were invited to participate in the study.

- A pre-validated questionnaire from the Medical Research Council in Cape Town was used with written permission of the authors (Becker, Bester, Reyneke, Labadarios, Monyeki \& Steyn, 2004:63-69).

- $\quad$ Structured interviews, using the questionnaire as the basis, ensured structural coherence throughout all the interviews.

- $\quad$ Face to face interviews ensured understanding of the questions as the fieldworkers acted as interpreters and answered any questions the participants posed in their own language.

- $\quad$ Trained and supervised fieldworkers were used.

- Only one person, a registered professional nurse, took the physical measurements preventing inter-rater variability (Burns \& Grove, 2005:375).

- Internationally accepted cut-off points were used to categorise the physical measurements of the participants.

\section{Ethical considerations}

Approval to conduct the study was obtained from the Ethics Committee of Tshwane University of Technology (\# 2006/07/007); the Health Department of North West Province and the management of the three primary health clinics. The participants signed informed consent before participating in the study. All hypertensive adults entering the day clinic for management of hypertension in the data-gathering period were invited to participate and were included in the study. The data gathering was completed in a separate room to guarantee privacy and anonymity, with confidentiality being ensured by numbering the questionnaires sequentially.

\section{Results of the study}

General and demographic profile More women $(n=78)$ than men $(n=23)$ participated in the study. The median of the sample was the 51-60 years age group constituting $29.7 \%$ of the group. The majority of the sample $(63.3 \%)$ had primary school education or less and in line with the advanced age group, $65.3 \%$ were pensioners.

\section{Personal medical history}

The most prevalent risk factors in the sample were high blood cholesterol $(34.7 \%)$ and diabetes mellitus (19.8\%). From family history, the prevalence of obesity, hypertension and diabetes ranged from $30.7 \%$ to $34.7 \%$.

Complementary alternative medications used for hypertension were investigated; Cape Aloe was most frequently used $(11.9 \%)$, followed by guava leaves (10.9\%). To assess compliance with their hypertension treatment, $31.7 \%$ admitted that they occasionally forgot to take their medication and when asked if they drank their medication the morning before attending the clinic, $18.8 \%$ reported in the negative.

\section{Knowledge about hypertension}

Several questions were posed to investigate the participants' knowledge relating to hypertension. The first two questions in the hypertension knowledge section focussed on non-pharmacological methods individuals can use to control hypertension. The first question focussed on methods generally available for individuals to reduce hypertension and the second focussed on what the participant could person- 
Table 1: Comparison between general and personal nonpharmacological hypertension control methods ( $n=101$ )

\begin{tabular}{|l|l|l|}
\hline Control methods & In general (\%) & Personal (\%) \\
\hline Control weight & 86.1 & 83.2 \\
Reduce salt intake & 93.1 & 92.1 \\
Reduce alcohol intake & 64.4 & 57.4 \\
Don't know at all & 12.9 & 2.0 \\
Do exercise & 67.3 & 72.3 \\
Home remedies & 10.9 & 9.9 \\
Traditional beer & 2.0 & - \\
\hline
\end{tabular}

ally do to control their own hypertension. Table 1 presents the results of the comparison between general and personal non-pharmacological hypertension control methods.

Table 1 provides evidence that knowledge does not necessarily lead to behaviour change. Although $86.1 \%$ knew weight control is critical in hypertension management, $83.2 \%$ still thought their own weight could be reduced. The same situation exists for reduction in salt intake; though nine out of ten knew that a reduction would lessen hypertension, they also indicated that they could decrease their own salt intake.

To test participants' knowledge and understanding of their hypertension, they were asked questions regarding what would happen to their health if the hypertension went untreated; $94.1 \%$ reported the occurrence of a stroke, with a heart attack or heart failure being reported by $42.6 \%$. In addition, in terms of their current level of hypertension control, most $(70.3 \%)$ reported it was normal, with $26.7 \%$ reporting it was low. No one reported their blood pressure level to be high although $30.7 \%$ had uncontrolled hypertension. Of concern is that the participants with uncontrolled hypertension were less knowledgeable regarding the complications of stroke $(28.7 \%)$ compared to those with normal blood pressure $(39.4 \%)$.

\section{Habit and lifestyle changes}

Participants' perceptions regarding their own weight were explored and of the group who were overweight (BMI Z" $\left.25-29.9 \mathrm{~kg} / \mathrm{m}^{2}\right)$, only $22 \%$ perceived themselves to be so. The participants were also asked whether they thought being overweight had an influence on their level of hypertension and the majority $(93.1 \%)$ agreed.

Diet was investigated extensively in terms of both salt usage and healthy food choices. Only $4 \%$ of the participants reported eating very salty food, with $85 \%$ indicating lightly salted food. The knowledge of the amount of salt to be added daily to the food of hypertensive persons was also investigated. A small group $(20.8 \%)$ reported no salt should be added, with the majority $(75.2 \%)$ indicating half a teaspoon of salt.

To test the participants' knowledge of food choices for overweight, hypertensive people, they were given a list of healthy and unhealthy food choices (Table 2).

The participants' knowledge of healthy food choices (Table 2) included soft maize porridge $(58.4 \%)$ but the surprising result in Table 2 is the 'Do not know' column. A number of choices are clearly not healthy food options for overweight and hypertensive persons. Participants were unaware that pastries (45.5\%), Cremora ${ }^{\Phi}(52.5 \%)$, cheese and cheese spread $(48.5 \%)$, Bovril $^{\mathbb{D}}(42.6 \%)$, vetkoek (61.4\%), Fondor ${ }^{\text {s }}$ or Aromat ${ }^{\text {}}$ $(62.4 \%)$ and samoosas $(64.4 \%)$ indicate a risk in terms of weight and hypertension control.

Participants' knowledge was further tested, regarding healthy diet, by posing several true or false questions ( $\mathrm{Ta}$ ble 3).
An encouraging fact was that $83.2 \%$ of participants agreed that vegetables and fruit can reduce hypertension (Table 3 ). with six in ten (63.4\%) agreeing that herbs can be used instead of salt to flavour food. More alarming is that $57.4 \%$ of the participants believed tinned, cured and smoked meat had a low salt content and $26.7 \%$ indicated that Aromat $($ and Fondor $B$ contains no salt.

An interesting result from Table 3 is the unsure column. Fewer participants were unsure about their answers when they had to indicate a healthy food choice. When it was stated that Aromat ${ }^{*} /$ Fondor $^{x y}$ do not contain salt, $5.9 \%$ were unsure and $67.3 \%$ disagreed and indicated they did know. In contrast, when asked if Aromat ${ }^{\circledR}$ or Fondor $B$ is a healthy food choice, $64.2 \%$ were unsure and $35.6 \%$ indicated negatively. The knowledge that the product contains salt is not linked to a healthy food choice. Combined with this dichotomy is the proportion $(92.1 \%)$ who indicated they eat their food lightly salted. The question may be asked "what is the interpretation of 'lightly' salted in this sample of hypertensive patients?"

The distribution of physical activity is divided in terms of commuting activity, occupational and leisure time activity. Approximately one third of participants were physically inactive across all three activity categories. The proportion of the participants who could be classified as active or very active was low (commuting activity $27 \%$; occupational activity $10 \%$; leisure activity $9 \%$ ). This result may not be unexpected if the advanced age of the sample is taken into account.

Tobacco use as a risk factor for hypertension was also investigated with the majority $(87.1 \%)$ having never smoked.

\section{Life threats}

Two questions examined the participants' perceptions regarding their vulnerability due to hypertensive status. The first question dealt with the possible risks the participants are exposed to if they do not adhere to treatments as planned. The majority $(59.4 \%)$ chose the extremely dangerous option. The second question asked what the participants thought they may die of (life threats) and each had to indicate three possibilities. More than half $(67.3 \%)$ of 
Table 2: Healthy food choices for overweight and hypertensive people ( $n=101)$

\begin{tabular}{|c|c|c|c|c|c|c|}
\hline \multirow[b]{2}{*}{ Food items } & \multicolumn{2}{|c|}{ Yes } & \multicolumn{2}{|c|}{ No } & \multicolumn{2}{|c|}{ Do not know } \\
\hline & $\mathbf{n}$ & $\%$ & $\mathbf{n}$ & $\%$ & $\mathbf{n}$ & $\%$ \\
\hline Fried eggs & 42 & 41.6 & 29 & 28.7 & 30 & 29.7 \\
\hline Fruit e.g. apples and oranges* & 94 & 93.1 & 5 & 5.0 & 2 & 2.0 \\
\hline Pastries & 15 & 14.9 & 40 & 39.6 & 46 & 45.5 \\
\hline Chocolate & 4 & 4.0 & 58 & 57.4 & 39 & 38.6 \\
\hline Tinned meat, fish or chicken & 36 & 35.6 & 34 & 33.7 & 31 & 30.7 \\
\hline Samp and beans* & 62 & 61.4 & 15 & 14.9 & 24 & 23.8 \\
\hline Cremora ( & 21 & 20.8 & 27 & 26.7 & 53 & 52.5 \\
\hline Vegetables (broccoli, spinach)* & 80 & 79.2 & 10 & 9.9 & 11 & 10.9 \\
\hline Cheese and cheese spread & 12 & 11.9 & 40 & 39.6 & 49 & 48.5 \\
\hline Chips & 17 & 16.8 & 48 & 47.5 & 36 & 35.6 \\
\hline Liver & 33 & 32.7 & 37 & 36.6 & 31 & 30.7 \\
\hline Brown bread* & 72 & 71.3 & 16 & 15.8 & 13 & 12.9 \\
\hline Tinned or packet soup & 43 & 42.6 & 23 & 22.8 & 35 & 34.7 \\
\hline Bovril $^{\infty}$ & 16 & 15.8 & 42 & 41.6 & 43 & 42.6 \\
\hline Soft maize porridge* & 59 & 58.4 & 17 & 16.8 & 25 & 24.8 \\
\hline Vetkoek & 2 & 2.0 & 37 & 36.6 & 62 & 61.4 \\
\hline Fondor $^{\circledR 8}$ or Aromat ${ }^{(8)}$ & 2 & 2.0 & 36 & 35.6 & 63 & 62.4 \\
\hline Samoosas & 0 & 0 & 36 & 35.6 & 65 & 64.4 \\
\hline
\end{tabular}

* Healthy food choices

the participants feared a heart attack, $49.5 \%$, (almost half) thought of AIDS, $44.6 \%$ indicated hypertension, followed by diabetes $(40.6 \%)$. Interestingly, no mention was made of a stroke.

\section{Physical measurements}

More females $(57.7 \%)$ than males $(21.7 \%)$ were overweight or obese. Seventy-two percent of the females having uncontrolled hypertension $(n=25)$ were overweight or obese. The participants' hip-to-waist ratio was measured and again the females had the highest risk. The majority of females $(75.6 \%)$ and one third of males (34.8\%) had a hip-to-waist ration above the cut-off point, which is $\geq 1.0$ for males and $\geq$ 0.85 for females (Department of Health, 2002).

\section{Summary of evidence generated through the study}

As hypertension control is the ultimate purpose of this study, evidence of factors playing a role in the study sample are summarised.

- Female gender: more females $(32.5 \%)$ than males $(26.1 \%)$ had uncontrolled hypertension.

- Age: More females, less than 40 years of age, had hypertension compared to younger males.

- Educational level: The sample had a lower educational level than that reported in the 2001 census.

- Pre-existing conditions:

Known cardiovascular risk factors in the study sample were high blood cholesterol (34\%) and diabetes mellitus (20\%).

- Family history: A positive family history was discovered for cardiovascular disease specifically on the maternal side of the family.

- Compliance with treatment: Variable responses to questions investigating compliance to taking medication as prescribed. 
Table 3: Results of true or false questions $(n=100)$

\begin{tabular}{|c|c|c|c|c|c|c|}
\hline Indicate whether true or false & $\begin{array}{l}\text { True } \\
\text { n }\end{array}$ & $\%$ & $\begin{array}{l}\text { Fals } \\
\text { n }\end{array}$ & $\%$ & $\mathbf{n}^{\mathbf{t}}$ & $\begin{array}{l}\text { Ire } \\
\%\end{array}$ \\
\hline Fresh fish is high in salt & 12 & 11.9 & 80 & 79.2 & 9 & 8.9 \\
\hline Aromat $\circledast$, Fondor $\circledast$ and stock cubes do not contain salt & 27 & 26.7 & 68 & 67.3 & 6 & 5.9 \\
\hline Vegetables and fruit can reduce your hypertension* & 84 & 83.2 & 13 & 12.9 & 4 & 4.0 \\
\hline Tinned, cured and smoked meat has low content of salt & 58 & 57.4 & 29 & 28.7 & 14 & 13.9 \\
\hline Herbs can be used to flavour foods instead of salts and other spices* & 64 & 63.4 & 25 & 24.8 & 12 & 11.9 \\
\hline
\end{tabular}

* Statements are true

- Knowledge: The group with uncontrolled hypertension was less knowledgeable regarding the complication of stroke (28.7\%) compared to the normal blood pressure group (39.4\%) and the low blood pressure group (31.9\%). A general lack of knowledge was revealed regarding a healthy diet as participants do not recognise the concept 'healthy food choice' and this concept should not be used when investigating knowledge regarding food.

- Body image and weight: Participants' perceptions regarding their own weight and physical activity did not correspond with their actual BMI categories and levels of physical activity. Their perception erred to the underweight side rather than the overweight/obese side.

- Complications of hypertension: Though the participants knew stroke to be a complication of hypertension, none considered they could die of this.

\section{Discussion of the results}

The Health Promotion Model (Pender, Murdaugh \& Parsons, 2002:60) indicate that individual characteristics and experiences involve prior related behaviour and personal factors.

\section{Perceived body mass}

One such factor is the perception disparity between a person's perceived and measured or actual weight also reported by Reddy (2004:182). The potential problem of perceiving to have a normal weight is that all health education regarding diet will be ignored as not being applicable. The influence of perception of having normal weight will negate the perceived benefit of dieting as this has apparently already been achieved. The commitment to the dieting plan of action will be low (Pender, Murdaugh \& Parsons, 2002:60).

To emphasise the participants' lack of awareness of their risk of cardiovascular disease in terms of their body size, the waist circumference is relevant. According to the results, $66.3 \%$ had a waist circumference above the cut off point. The implication of abdominal adiposity is that excess adipose tissue releases several products that exacerbate hypertension and other cardiovascular risk diseases (Scott \& Cohen, 2004:3). Clinical trials have proven that weight loss is effective in the primary prevention of hypertension, plus reduction of both diastolic and systolic pressure (Dickey \& Janick, 2001:392).

The participants' physical measurements also indicated increased risk. The majority had a waist-to-hip circumference above the cut off point, a known risk for cardiovascular disease. Even though the participants knew weight was a risk factor for hypertension and with weight reduction it can be controlled, the knowledge did not result in a change of behaviour. The waist alone is a good measurement for intra-abdominal fat (Gaw \& Lindsay, 2004:167).

The authors state that BMI alone does not take fat distribution into account, so it is useful to measure obesity with waist-to-hip ratio and BMI.
Knowledge of nutritional factors

The study provided evidence that the participants were uninformed regarding a healthy diet for a hypertensive person and if knowledgeable, they did not apply the information to improve and maintain their health. The lack of transition from knowledge to a change in behaviour maintains hypertension patients' risk level. Frequently, the change would be minor but the risk reduction significant. Several authors (Hooper, Bartlett, Smith \& Ebrahim, 2003; He \& McGregor, 2004) report a modest reduction in sodium for at least four weeks resulted in significant reduction in blood pressure. The authors suggest a daily intake of sodium from 3-12 g/day to achieve a long-term reduction of strokes, heart attacks and heart failure.

Another implication of lack of knowledge is that the participants use unhealthy foods whilst being hypertensive. Lack of knowledge in this instance also acts as a situational influence, which will affect the participants' commitment to a plan of action (Pender, Murdaugh \& Parsons, 2002: 60). Becker et al. (2004:67) also found that the knowledge of diet and hypertension to be poor. The results of the study indicated that although the participants have been educated about healthy food for hypertension, they still lack information as to which food is healthy and continue to consume a diet familiar to them though not recommended for hypertensive people.

\section{Knowledge of complications of hypertension}

An interesting result was the compli- 
cations that could occur if hypertension is not treated. The participants were knowledgeable about hypertension complications: $94.1 \%$ reported stroke, $42.6 \%$ reported heart attack and $22.8 \%$ reported kidney failure though none thought they would die of a stroke. According to the Health Belief Model, beliefs about personal susceptibility and the seriousness of a specific illness combine to produce the degree of threat or negative valence of that illness (Pender, Murdaugh \& Parsons, 2002:37). Dennison, Peer, Lombard, Kepe, Levitt, Steyn and Hill (2007:484) support the findings of the study in that black South Africans do not perceive untreated hypertension as serious.

\section{Risk factors}

In terms of personal and family medical history, evidence that could influence nursing practice was generated. The participants were older adults, those above 50 years of age constituted $75.5 \%$ of the sample. According to Gaziano, Steyn, Cohen and Opie (2005:3569), hypertension is the most prevalent risk factor for vascular disease in older adults affecting $60 \%$ of adults 60 years or older. In a study by Franco, Peeters, Bonneux and De Laet $(2005: 282)$, the authors illustrate that irrespective of gender, 50 year old hypertensive persons had a shorter life expectancy than normotensive persons.

Other risk factors, high blood cholesterol $(34.7 \%)$ and diabetes mellitus $(19.8 \%)$ were discovered in the study sample. From family history, as reported by the participants, obesity, hypertension, diabetes and stroke were the common risk factors from their mother's side. According to Scott and Cohen (2004:2), hypertension and elevated low density cholesterol are common risk factors for metabolic syndrome and therefore the underlying risk factors for cardiovascular diseases. Family history alone is a risk factor for most chronic diseases and can assist in early detection and be used in preventative intervention strategies (Yoon, Scheuner \& Khoury, 2003:128).

\section{Limitations}

One urban area, Ga-Rankuwa was chosen for the purpose of the study. Due to the convenient sample, the results cannot be generalised. The data gathered was self-reported and subject to both recall and social desirability bias. The data-gathering instrument used was a questionnaire that offered no open-ended questions and therefore not possible to gain a deeper understanding of concepts, for example 'lightly salted'.

\section{Recommendations}

Several recommendations emanate from the study.

In terms of nursing practice, healthy eating and healthy food choices should be a central pillar in teaching hypertensive adults non-pharmacological methods to maintain hypertension control.

- A national health campaign on the burden of hypertension would raise awareness of the disease because hypertension alone is a predisposing risk factor for stroke and other cardiovascular disease. Such a campaign should be initiated from the primary health care level as the reality of hypertension and the complications are experienced daily in clinics.

- Additional research is required to investigate concepts such as 'light salt use' from the hypertensive person and the health care provider's perspective as well as from the various socio-cultural groups in South Africa.

- The content and method of health education given must be investigated and evaluated in terms of long-term behaviour change in the recipients. Additionally, intervention studies for the management of hypertension within the community must be developed, implemented and evaluated for a positive outcome, namely hypertension control.

\section{Conclusion}

Hypertension is among the most prevalent and important risk factors for cardiovascular, cerebrovascular and renal disease. Hypertension can only be controlled with good medication adherence and deliberate long-term changes in a person's lifestyle. Achieving these changes, however, has proven to be elusive and failure is frequent. Community-specific interventions, based on evidence generated from that community, may be the only way to reduce the burden of hypertensive disease.

\section{References}

BALES, CW \& RITCHIE, CS 2004: Handbook of clinical nutrition and aging. New Jersey: Human.

BECKER, H; BESTER, M; REYNEKE, N; LABADARIOS, D; MONYEKI, K D; \& STEYN, NP 2004: Nutrition related knowlegde and practices of hypertensive adults attending hypertensive clinics at day hospitals in the Cape Metropole.Curationis. 27: 63-69.

BURNS, N \& GROVE, S 2005: The practice of nursing research: conduct, critique and utilisation. Philadelphia: Saunders.

GAW, A \& LINDSAY, G 2004: Coronary heart disease prevention: a handbook for the health care team. Philadelphia: Lippincott.

HE, F \& MACGREGOR, G 2004: Effects of longer-term modest salt reduction on blood pressure Review. Cochrane Database Systematic Reviews. 3 .

HOOPER, L; BARTLETT, C; SMITH, DG \& EBRAHIM, S 2003: Reduced dietary salt for prevention of cardiovascular disease. Cochrane Database System Review. 2 .

IJUMBA, P; DAY, C \& NTULI,A 2004: South African Health Review. (In HEALTH eds. 2003/04.Durban: Health System Trust.)

LOBIONDO WOOD, G \& HABER, J 2006: Nursing research: methods and critical appraisal for evidence based practice. St Louis: Mosby.

PELTZER, K 2001: Knowledge and attitudes of primary care nurses and midwives towards health promotion in rural South Africa. Curationis 4: 52-58.

PELTZER, K 2002: Health behaviour among black and white South Africans. Roval Society for the Promotion of Health 122: 187-193. 
PENDER, N; MURDAUGH,C \& PARSONS, A 2002: Health Promotion in Nursing Practice. Julie Alexander: Prentice hall.

REDDY, P 2004: Chronic diseases. Durban: Health System Trust.

SMITH, S 1999: An introduction to the curative aspects of primary health care. Glenstantia: GM printers.

DEPARTMENT OFHEALTH(SOUTH AFRICA) 2003: South African standard treatment guidelines and essential drug list for primary health care: South Africa. 3rd ed. Pretoria: National Department of Health.

STATISTICS SOUTH AFRICA 2001: Census 2001 figures for $\mathbf{G a -}$ Rankuwa. http://www.statssa.gov.za/ extract.htm.Accessed 18/03/2006].

STEYN, K 2005: Hypertension in South Africa. (In STEYN, K; FOURIE, JM; \& TEMPLE, N, eds. 1995-2005: Chronic diseases of lifestyle in South Africa. Cape Town.)

VAN ROOYEN,JM; HUISMAN, HW; ELOFF, FC; LAUBSHER, PJ; MALAN, L; STEYN, HS \& MALAN, NT 2002: Cardiovascular reactivity in Black South African males of different groups: the influence of urbanisation Ethnicity and Disease 12: 69-75. 